\title{
Analysis of the Protective Effect of NutriSim (C) and the Inflammatory Response in a Model of LPS-Induced Shock from E. coli Serotype 0111:B4
}

\section{Daniela L Delgado-Lara', José J Hernández-Cruz ${ }^{1,2}$, Luís A Romero- Tirado², Rolando Roméro-Dávalos², José J Hernández-Andalón², Irma E Velázquez-Brizuel and Genaro Gabriel Ortiz ${ }^{1 *}$}

${ }^{1}$ Department of Philosophical and Methodological Disciplines, University Center of Health Sciences, University of Guadalajara, Guadalajara, Mexico

${ }^{2}$ Laboratories BioSim (C); Scientific Research and Development Area, MXCD, Mexico

*Corresponding Author: Genaro Gabriel Ortiz, Department of Philosophical and

Methodological Disciplines, University Center of Health Sciences, University of

Guadalajara, Guadalajara, Mexico.
Received: April 15, 2020

Published: May 21, 2020

(C) All rights are reserved by Genaro Gabriel Ortiz., et al.

\begin{abstract}
The term septic shock or septicemia were considered analogous to endotoxic shock or endotoxicosis and used to designate the state of acute metabolic-circulatory dysfunction, which is trigger after the release into the body of bacterial endotoxins, secondary to an initial septic process, or subsequent to experimental administration of isolated endotoxins, respectively. Despite the extensive use of the term "septic shock" in clinical and preclinical studies to refer to this pathological state, we will now refer to this state only as sepsis or LPS-induced sepsis as appropriate.

Experimental animal models treated with isolated bacterial endotoxins replicates many of the hematologic, hemodynamic, and metabolic abnormalities characteristic of patients with bacterial infections that trigger shock. Endotoxins are macromolecular complexes made up of lipopolysaccharides (LPS) and proteins located on the outer membrane of Gram-negative bacteria.

The main pro-inflammatory cytokines are tumor necrosis factor-alpha, interleukin 1, interleukin 6 and interleukin 8. The expression of these cytokines is responsible for many of the clinical effects and lesions observed in LPS-induced sepsis. LPS is a potent "stimulator" of the immune response, inducing cytokine production in macrophages and B-cells.

The present work aimed to study the effect of Nutrisim $(C)$ on inflammation in an in vivo model of sepsis-induced by Escherichia coli 0111: B4 lipopolysaccharide. We found that the nutritive supplement NutriSim has shown a decrease in pro-inflammatory cytokines and even a protective effect against LPS. Further clinical research has to be made.

Keywords: NutriSim C; Lipopolysaccharides; Inflammation; Endotoxic Shock
\end{abstract}

\section{Abbreviations}

LPS: Lipopolysaccharides; LBP: Lipopolysaccharide-Binding Protein; BGN: Enteric Gram-Negative Bacilli; KDO: 2-Keto-3-Deoxyoctanoic Acid; GPI: glycosylphosphatidylinositol; sCD14 Soluble Cluster of Differentiation 14; mCD14: Membrane Cluster of Differentiation 14; TLR4: Toll Like Receptor 4; NFKB: Nuclear Factor Kappa B; TNFa: Tumor Necrosis Factor Alpha

\section{Introduction}

When speaking of shock, we define a life-threatening health condition that follows any type of aggression against the body and is characterized by a decrease in blood flow, tissue hypoxia and consequently, alteration of cellular metabolism. This continuous series of pathological manifestations, progressively more serious than its predecessor, has been classified as reversible or moderate and irreversible or severe, taking into account the characteristics of vascular, cellular, and metabolic phenomena. When homeostatic mechanisms cannot counteract these disturbances, a series of decompensatory processes take place that are detrimental to recovery and cause a state of irreversible shock. From the etiological point of view, shock is not a unique syndrome, but under this term, a series of alterations that appear in cases of severe bleeding, myocardial infarction, cardiac tamponade, generalized pulmonary embolism, trauma, burns, and bacterial infection are included; each type of shock is classified according to its etiology as hemorrhagic, cardiogenic, traumatic neurogenic and septic [1]. The cur- 
rent definition of this life-threatening organ dysfunction caused by a dysregulated host response to infection should be called sepsis, which designates the body's response to infection that collaterally injures its tissues and organs. Therefore, if not recognized, it evolves through time; additionally, we must keep in mind that this response depends on pathogen virulence and host factors to determine the damage [2].

Nowadays, septic shock is a subset of sepsis and designates the state of acute metabolic-circulatory abnormalities enough to increase mortality, and it is characterized by clinical construct of sepsis with persistent hypotension requiring vasopressors to maintain mean arterial pressure $>65 \mathrm{~mm} \mathrm{Hg}$ and having a serum lactate level $>2 \mathrm{mmol} / \mathrm{L}$ despite adequate volume resuscitation [2]. In this paper, we will use the terms agreed in the third international consensus on sepsis and septic shock (sepsis-3), which were specifically made to avoid confusion between the existing terms to determine this pathological state. Despite the extensive use of the term "septic shock" in clinical and preclinical studies to refer to it, we will now refer to this state only as LPS-induced shock. The symptoms of sepsis include fever, hypothermia, tachycardia, weak pulse, oliguria or significant edema, hypotension, alteration of mental status, hyperglycemia, among others [3]. These are consequences of tissue hypoperfusion (low contribution of fluids and oxygen) that cause cellular suffering and, therefore, multiorgan failure. The physiological response is manifested in a series of pathological alterations that affect practically all organs, with variations in hemodynamic parameters, hematologic, cardiovascular-pulmonary, gastrointestinal tract, nervous system, skeletal muscle, endocrine systems, and renal factors [4]. Sepsis is the leading cause of death in critically ill patients. Septic shock appears in $10.4 \%$ of patients at ICU admission, reaching crude mortality associated with $46.5 \%$ in patients who met the diagnostic criteria for sepsis-3, this fact highlights the need to find and use all available therapeutic resources [5,6]. Experimental treatment on animal models with purified bacterial endotoxins replicates many of the hematologic, hemodynamic, and metabolic abnormalities characteristic of patients with sepsis. It is widely accepted that endotoxins can cause such immune response that can trigger sepsisinduced state identical to sepsis and can be used for investigation on animal models [7].

\section{Endotoxins and lipopolysaccharides and immune response}

Endotoxins are macromolecular complexes made up of lipopolysaccharides (LPS) and proteins, which are released into the bloodstream during lysis or periods of active growth of Gram-negative bacteria (BGN). Enteric BGN causes the highest number of cases of sepsis, as well as in specific contexts non-fermenting ba- cilli such as Pseudomonas aeruginosa and more rarely aerobic BGN, such as Bacteroides fragilis and Legionella pneumophila [8]. The terms endotoxin and LPS are used interchangeably since LPS possesses all the biological activities of endotoxins, although it is not the only one endotoxin. For this reason, purified LPS is commonly used in experimental studies [8]. Lipopolysaccharides (LPS) are the major components of the outer membrane of Gram-negative bacteria such as E. coli, Klebsiella pneumoniae and S. typhimurium; Bacterial LPS are the main target of antibodies produced by the immune system in response to bacterial infection [9].

\section{Chemical structure of the lipopolysaccharides}

LPS consists of a hydrophilic portion of oligosaccharides attached to a hydrophobic lipid portion called Lipid A. These oligosaccharides do not have endotoxic activity, but they possess immunostimulatory properties [10].

LPS toxicity resides in the next portion [11], Which consists of two parts: O-specific chain and basal nucleus:

- $\quad$ O-specific side chain: Made up of a variable number of oligosaccharides formed in turn by 3 or 4 different units of sugars. The monosaccharide sequence is characteristic of each bacterial species and therefore is the carrier of the serological specificity of each LPS [12].

- Basal nucleus: Much shorter and more homogeneous than the $\mathrm{O}$-antigen, it is divided into two regions, an external one, normally composed of glucose, galactose and $\mathrm{N}$-acetylglucosamine and an internal one made up of 2-keto-3-deoxyoctanoic acid (KDO) and heptoses. This inner core frequently contains phosphate residues [13] (Figure 2).

Lipid A is the most conserved part of the LPS molecule [14], it counts with a similar structure and composition in many Gramnegative bacteria. The common structure is a $\beta$-D-glucosaminyl- $\alpha$ D-glucosamine disaccharide, phosphorylated at the 4' position of the glucosaminyl residue, and position 1 of glucosamine [15]. Also, positions 2 and 3 are substituted with 3-hydroxyacyl groups. Both phosphates and lipid residues are essential for the biological activity of the molecule (Figure 1) [16].

\section{Immune response to lipopolysaccharides}

Bacterial LPS induces pleiotropic activation of the immune system, which subsequently results in LPS induced-shock [17]. The LPS 1) Binds directly to caspases 4 and 5, which causes their activation and lead monocytes, endothelial cells, and keratinocytes to death [18]. 2) Activate complement through classical and alternative 


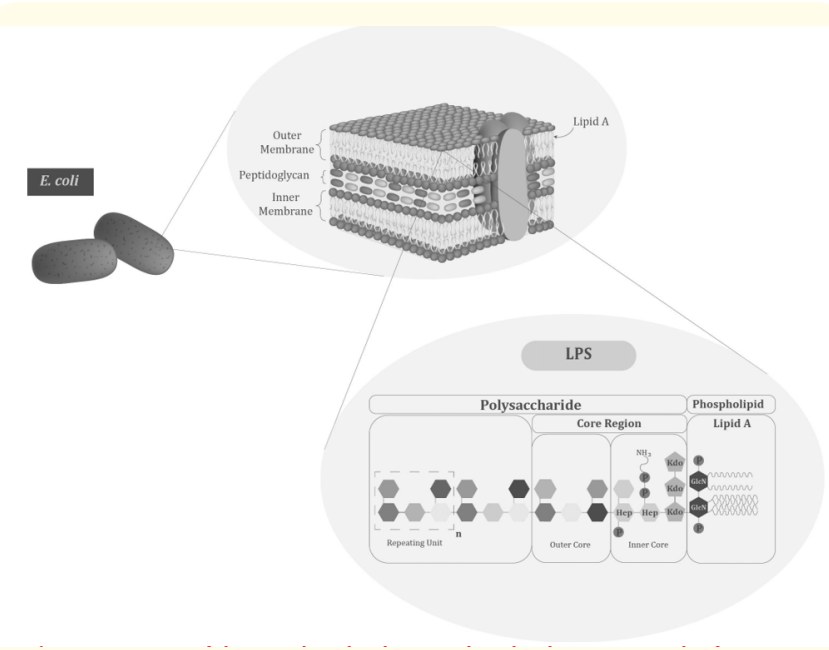

Figure 1: Structure of the Lipopolysaccharides. Lipopolysaccharides consists mainly of two parts: a glucolipid called lipid $\mathrm{A}$, and a heteropolysaccharide called the core region linked by 2-keto-

3-deoxyoctanate acid sugar (KDO).

pathways, provoking opsonization to facilitate phagocytosis [19]. 3) Binds to CD14, which is a glycosylphosphatidylinositol-bound or glycoprotein expressed in monocytes, macrophages, and neutrophils. CD14 has two main isoforms: the membranous isoform (mCD14) and the soluble isoform (sCD14) [20]. 4) Binds to the TLR4-MD2 complex that leads to the innate and adaptive immune response [21]. Upon entering the circulation, LPS rapidly binds to LBP (LPS binding protein), which is an acute phase glycoprotein of approximately $60 \mathrm{kDa}$. Forming the LPS-LBP complex [22], LBP acts as an antibody that attracts particles that carry LPS bound to them, and this complex is quickly recognized by the mCD14 receptor on monocytes and macrophages; mCD14 is a differentiation antigen of monocytes that binds to the LPS-LBP complex-forming LPS-LBP-CD14 complex [23]. Isoform mCD14 induces phagocytosis activation and cytokines production fundamentally TNF- $\alpha$, IL-1, IL-6, IL-8, and IL-12 on macrophages [24]; sCD14 can be released from the cell surface and binds to LPS. Afterward, the LPSLPB complex binds to the TLR4-MD2 complex, which triggers the synthesis and almost immediate release of TNF- $\alpha$ by monocytes. Moreover, LPS-TLR4-MD2 complex actives mainly pathways NFאB to mount the immune response by inducing B7 family molecules required for activation of naive $\mathrm{T}$ cells by antigen-presenting cells [21], being a critical link between pathogen detection and the induction of the adaptive immune response (Figure 2) [25].

\section{Inflammation and shock}

Macrophages are probably the most important target cells for endotoxins in the development of an endotoxicity picture; after

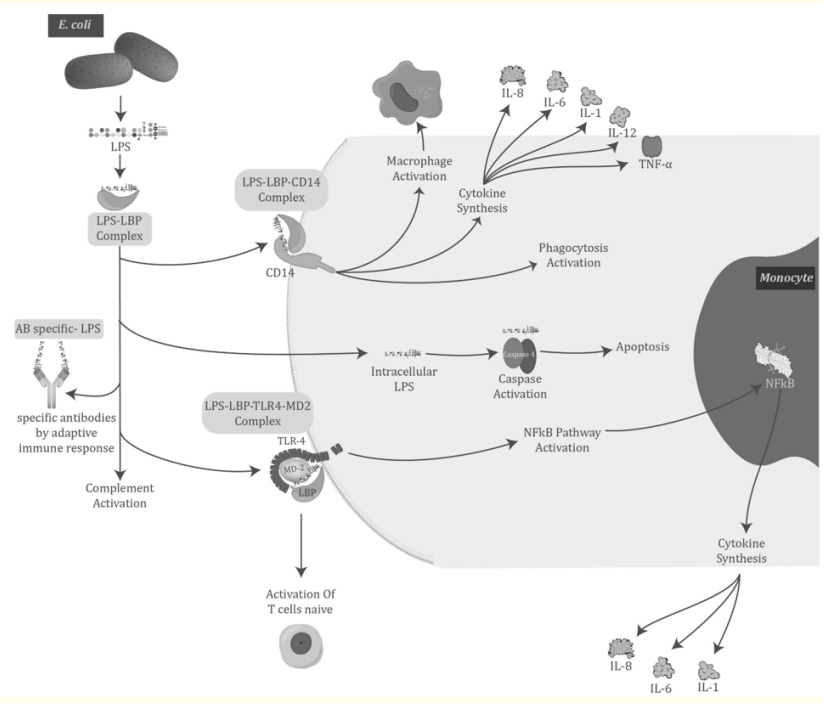

Figure 2: Immune response to Lipopolysaccharides.

interaction with the bacterial toxin, the macrophage is activated and begins to produce a large number of specific cytokines, including $\mathrm{TNF} \alpha$, which together with IFN $\gamma$ are the primary mediators of endotoxin activity [26]. LPS also induces monocyte activation in a multistep process resulting in cytokine production. Lymphocytes also respond to LPS in nanogram concentrations and secrete cytokines like TNF $\alpha$ itself. Bacterial LPS induces pleiotropic activation of the immune system, which subsequently results in sepsis [27]. Cytokines such as TNF $\alpha$, IL-1, IL- 6 , and IL-8 are capable of mediating a wide range of biological effects; these effects include stimulation of antibacterial function, mobilization of sources of substrates to preserve cellular function [28]. This entire inflammatory cascade is designed to preserve and protect the host; however, some of these mediators are capable of inducing adverse effects, and the cascade of humoral and cellular mediators may become temporarily independent of the initial infection that caused it and thus result in multi-organic systemic failure and cause the death of the host [29]. For this reason, the study of new approaches to the treatment of shock is a topic of medical interest for several pharmaceutical companies (Figure 3) [30].

\section{Treatment}

The treatment of sepsis is currently focused on two points of view: the etiological treatment, with little or no clinical repercussion, which seeks different strategies that can modulate the activated mediator battery and, consequently, stop the exaggerated inflammatory response, and the life support treatment. Today the healthcare reality of hospitals is based on GRADE system principles, which assess the quality of evidence from high to very low 


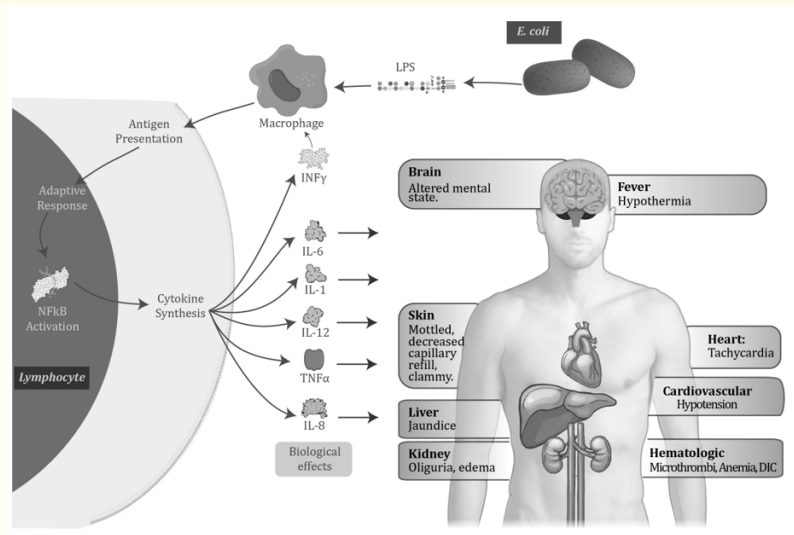

Figure 3: Adverse effects after immune cascade.

and determine the strength of the current recommendations. In essence, focuses on four aspects: the first consists of infection control with the early administration of appropriate antibiotics and the elimination of septic foci (drainage of abscesses, removal of contaminated catheters and prostheses); the second, in control cardiovascular hemodynamics through fluid therapy and the use of inotropic and vasoactive drugs; the third, to seek control of blood, which not rarely requires the use of artificial respirators, oscillatory ventilation and finally; the fourth, to provide an adequate nutritional supply $[2,31,32]$. The results of clinical trials applied to the treatment of sepsis in humans have not been effective; without a doubt, what seems most important of this is the inadequate selection of the patients in the elaboration of the groups that will be part of the trials [33]. It does not seem coherent to include patients based only on clinical criteria such as the presence of fever, leukocytosis, tachypnea, or tachycardia, essentially those that constitute the definition of sepsis. Indeed, even though all of them are present in two patients, it would be logical to consider them in entirely different watertight groups when the first, for example, suffered from a gram-negative infection, and in the second, a gram-positive infection. We would find a similar situation when the first one would have a biochemical pattern of pro-inflammatory mediators, and the second would have an anti-inflammatory who prevails. Thus, we do not attend to homogeneous groups, the number of patients to be included in the trials will be less, but of course, the results will be more reliable and therefore of greater consideration [33]. On the other hand, it is crucial to know when to start treatment and especially antibiotics; the antagonists of inflammatory mediators have been effective, especially when they were given before the onset of sepsis [34]. At the end of the day, anti-inflammatory and oxidative stress strategies will also be effective when the ap- propriate drug is applied, in the appropriate patient, in the right dose and at the right time [35].

Finally, we should ask ourselves:

1. Do we know sufficient about the etiopathogenic mechanisms of sepsis to affirm that our investigations are on the right track?

2. Is it true that the rampant inflammatory cascade is the real promoter of organ failure? Or there are some mechanisms that we do not understand yet?

3. How outstanding is the balance between pro-inflammatory mediators and anti-inflammatory mediators?

4. What is the relationship between oxidative stress in this process and whether it is worth intervening?

The answer to such questions will be the basis for future therapeutic applications.

\section{Effects of Nutrisim $\subset$}

Nutrisim (C) is a nutritive supplement made up of a mixture of amino acids of low molecular weight, minerals, and salts that are necessary for the right function of the human body: Arginine, Choline, Lysine, Magnesium, Calcium, and Ammonium Chloride [38]. The mechanism of action by which this supplement exerts its beneficial effect is still unclear. However, some studies had proven its efficacy in vivo by decreasing oxidative stress caused by ischemiareperfusion, the inhibition of pro-inflammatory cytokines, diminishing clinical symptoms due to LPS damage and increases survival in murine models [39-43].

In one study on lipopolysaccharide-treated male Wistar rats, they were randomized into five groups: control groups receiving a single intraperitoneal injection of physiologic saline solution or NutriSim (10 $\mu \mathrm{l} / 100$ g), respectively; LPS (20 mg/Kg, i.p. (LD100)); and NutriSim $@$ treatment 15 min before or after LPS in the same dose mentioned above. The results showed that a single dose of NutriSim @ prior to or before the endotoxic insult diminishes the production of serum TNF-a, IL-1, and IL-6 significantly (Figure 4) [43]. Also, in another work where the IL-4, IL-10, and oxide nitric were studied in an in vivo model, they divided into the same five groups with the same doses, NutriSim (C) given in a single dose decreased the production of the cytokines and oxide nitric catabolites (nitrates and nitrites) significantly. This demonstrates the strong anti-inflammatory activity of the supplement. One important thing of NutriSim (C) in both studies was that it is administration, in the absence of LPS, does not alter the serum cytokines levels, which might suggest that this supplement does not exert a negative effect on the immune system [42]. 
A deficiency of dietary amino acids impairs immune functions, increasing the susceptibility to an infectious disease. Hence the importance of amino acids such as arginine, lysine, and choline in the immune response; they regulate the activation of $\mathrm{T}$ and $\mathrm{B}$ lymphocytes, cellular redox status, and production of cytokines [44] which can be cytotoxic substances in the pro-inflammatory process.
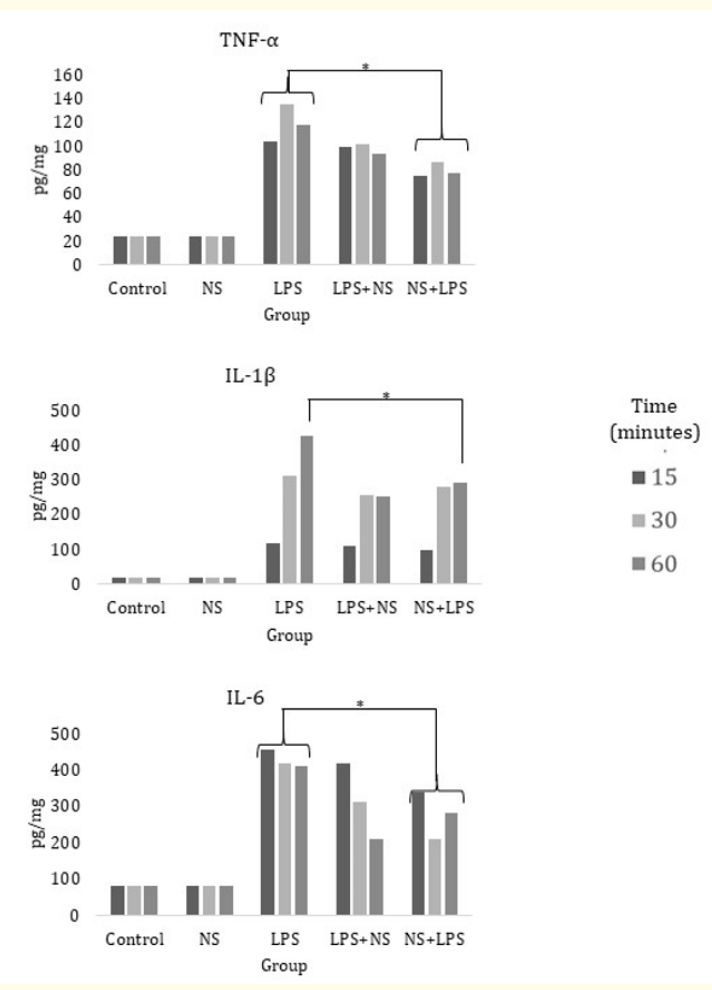

Figure 4: Effect of NutriSim (C) in cytokines. There was a statistically significant difference between the experimental groups as time passed in TNF and IL-6 ( $p<0.01$ ); treatment with NutriSim (C) after and before the injection of an LD100 of LPS diminished the serum IL-1 $\beta$ values significantly during the experiment $(\mathrm{p}<$ $0.05)$. Still, it was more evident at 60 minutes after LPS treatment.

- L-arginine, a semi-essential amino acid, has been described to play an important role in stress situations like sepsis, where the patients usually tend to show low levels of arginine amongst others like citrulline and ornithine $[45,46]$. Larginine has an important function in protein synthesis and subsequent conversion to a nitric oxide through the Nitric Oxide Synthase (NOS), which favors vasodilatation; it has been demonstrated that arginine diminishes lipid peroxidation in patients with diabetes mellitus [47] and plays versatile vital roles in nutrition and metabolism [48].
- Choline is a derivate of the amino acid serine; it has been demonstrated in vitro that choline inhibits TNF release in macrophages, and this might suggest that choline may have anti-inflammatory activity [49]. Choline is an important component of cell membranes, is the precursor of phosphatidylcholine and sphingomyelin, two structural phospholipids of biological membranes that are also precursors of intracellular messengers. Choline released from membrane phospholipids is important for the formation of the neurotransmitter acetylcholine, and also is important in the metabolism of methyl groups [50].

- $\quad$ Lysine forms part of the essential amino acids, which means that it must be obtained through the diet in adequate quantities to meet the body's needs. Lysine is required in the body for the manufacture of carnitine, and this is used for the proper metabolism of fats. It has shown that a restriction of lysine affects inflammatory responses in the kidney, liver, and spleen via mediating serum antibody volume, inflammatory cytokines, TLRs system, and ERK1/2 and NFKB signals in piglets [51]. Nevertheless, the physiological role of lysine extends beyond protein syntheses, such as reduced anxiety, inflammation, diarrheal morbidity, and muscle recovery in exercise or sarcopenia support [52].

Besides the amino acids contained in NutriSim $@$, it has salts and minerals, like Calcium, which helps the body to regulates the rhythm of the heart, eases insomnia, helps regulate the passage of nutrients in and out of cellular membranes, and assists in blood clotting, also, is very valuable in maintaining proper nerve and muscle function, as well as normal kidney function. Magnesium plays a crucial role in regulating the neuromuscular activity of the heart, maintaining normal heart rhythm, converting blood sugar into energy, and metabolizing calcium and vitamin C properly. Magnesium deficiency can result in calcium depletion, heart spasms, nervousness, confusion, muscular excitability, and kidney stones $[53,54]$. Altogether, this nutritive supplement could act at a cellular level ameliorating damage caused by oxidative stress and damage induced by endotoxins, and therefore increasing the survival rate. Previously, it was found in another study made by our group of research that $100 \%$ of rats treated with LPS died after 19 hours; $90 \%$ of the rats pre-treated with NutriSim@ died after 21 hours, but $80 \%$ of the rats who received NutriSim $@$ after LPS injection died 23 hours later (Figure 5); NutriSim (C) attenuated the damage caused by LPS, decreasing clinical symptoms, including shaggy hair, diarrhea, and prostration [40]. 
Considering that a diet low or free in this amino acids, like arginine, in patients with parenteral nutrition, produce hyperammonemia, metabolic acidosis or even coma, and also, arginine is capable of stimulating hormone secretion as insulin, glucagon catecholamines, prolactin and growth hormone, patients with the catabolic situation could have the benefit of this supplement. NutriSim (C), can modulate inflammatory response in an experimental model of endotoxic shock and have a protective effect in the presence of a compromised condition, increases the number of lymphocytes, stimulates rations of delayed hypersensitivity and increases the proliferative capacity of lymphocytes against mitogens and the activity of NK cells [55]. More important, in clinical trials, the use of arginine in patients with sepsis results in reduced whole-body protein turnover without affecting vasoactive medication [36] and has been used in critically ill patients like early enteral supplementation improving time for recovery of organ function compared with control [37].

\section{Conclusion}

LPS can stimulate an endotoxic shock liberating the main proinflammatory cytokines. NutriSim $@$ has shown to be effective in reducing the production of TNF alpha, IL-1, IL6, and IL-10 and increases the time of survival rate in rats with an injection of the supplement before or after LPS.

Our research group has found statistically significant changes at the cellular level regarding oxidative stress and immunomodulation, which have been reproduced by distinct approaches. With this framework and with evidence from clinical studies that have shown to improve clinical variables in the critically ill patient with other supplements that contain components similar to those contained in NutriSim $($ C), could it be pertinent to carry out clinical trials to assess whether the benefits observed by our group could it be reproduced in humans, including clinical and biochemical variables, with appropriate methodological studies, for the pathophysiological mechanisms in which it appears to be involved and could have the maximum benefit. It is also necessary to determine if this findings can be extrapolated on pathophysiological mechanisms with other diseases besides sepsis and establish histopathological changes and survival improvement in animal models as the main objective research variables in individuals treated with NutriSim $\subset$.

\section{Acknowledgements}

We want to thank the art designer Andrea Rosales for her collaboration in the elaboration of the figures in this article.

\section{Conflict of Interest}

The authors declare that there is no conflict of interest for the publication of this work.

\section{Bibliography}

1. Jean-Louis V., et al. "Circulatory Shock". New England Journal of Medicine 369.18 (2013): 1726-1734.

2. Mervyn S., et al. "The Third International Consensus Definitions for Sepsis and Septic Shock (Sepsis-3)". Journal of the American Medical Association 315.8 (2016): 801-810.

3. Snigdha J. "Sepsis: An Update on Current Practices in Diagnosis and Management". American Journal of the Medical Sciences 356.3 (2018): 277-286.

4. Jeffrey G., et al. "Sepsis: Pathophysiology and Clinical Management". British Medical Journal 353 (2016): 1-20.

5. Jean-Louis V., et al. "Frequency and Mortality of Septic Shock in Europe and North America: A Systematic Review and MetaAnalysis". Critical Care 23.1 (2019): 1-11.

6. Manu SH., et al. "Developing a New definition and Assessing New clinical Criteria for Septic Shock: For the Third International Consensus Definitions for Sepsis and Septic Shock (Sepsis-3)". Journal of the American Medical Association 315.8 (2016): 775-787.

7. Chris G., et al. "Synthetic and Natural Escherichia Coli Free Lipid A Express Identical Endotoxic Activities". European Journal of Biochemistry 148.1 (1985):1-5.

8. Steven O., et al. "Systemic Host Responses in Severe Sepsis Analyzed by Causative Microorganism and Treatment Effects of Drotrecogin Alfa (Activated)". Clinical Infectious Diseases 37.1 (2003):50-58.

9. Tim R., et al. "Cross-Specificity of Protective Human Antibodies against Klebsiella Pneumoniae LPS O-Antigen". Nature Immunology 19.6 (2018): 617-624.

10. James M., et al. "T CELL-INDEPENDENT". Annual Review of Immunology 13 (1995): 655-692.

11. Christian R et al. "Lipopolysaccharide Endotoxins". Annual Review of Biochemistry 71.1 2002): 635-700.

12. Yuriy K., et al. "Bacterial Lipopolysaccharides". Springer (2011): 41-115.

13. Tony C., et al. "A Gene Coding for 3-Deoxy-D-Manno-Octulosonic-Acid Transferase in Escherichia Coli: Identification, Mapping, Cloning, and Sequencing". Journal of Biological Chemistry 266.15 (1991): 9687-9696. 
14. Russell 0., et al. "Antibacterial Agents That Inhibit Lipid a Biosynthesis". Science 274.5289 (1996): 980-982.

15. Ernst R., et al. "Bacterial Endotoxin: Molecular Relationships of Structure to Activity and Function". The FASEB Journal 8.2 (1994): 217-225.

16. Christian A., et al. "Invited Review: Bacterial Lipopolysaccharides and Innate Immunity". Journal of Endotoxin Research 7.3 (2001): 167-202.

17. Girish R. "Gram-Positive and Gram-Negative Bacterial Toxins in Sepsis: A Brief Review”. Virulence 5.1 (2014): 213-218.

18. Jianjin S., et al. "Inflammatory Caspases Are Innate Immune Receptors for Intracellular LPS”. Nature 514.7521 (2014): 187-192.

19. Marina F., et al. "Interaction of Lipopolysaccharides and Lipid A with Complement in Rats and Its Relation to Endotoxicity". Infection and Immunity 19.3 (1978): 875-882.

20. Kitchens R. "Role of CD 14 in Cellular Recognition of Bacterial Lipopolysaccharides". Chemical Immunology 74 (2000): 61-82.

21. Beom SP., et al. "The Structural Basis of Lipopolysaccharide Recognition by the TLR4-MD-2 Complex". Nature 458.7242 (2009): 1191-1195.

22. Peter T., et al. "Lipopolysaccharide Binding Protein-Mediated Complexation of Lipopolysaccharide with Soluble CD14". Journal of Biological Chemistry 270.18 (1995): 10482-10488.

23. Haziot A., et al. "The Monocyte Differentiation Antigen, CD14, Is Anchored to the Cell Membrane by a Phosphatidylinositol Linkage". The Journal of Immunology 141.2 (1988): 547-552.

24. Maikel P., et al. "Lipopolysaccharide Regulates Macrophage Fluid Phase Pinocytosis via CD14-Dependent and CD14-Independent Pathways". Blood 93.11 (1999): 4011-4018.

25. Alan A., et al. "Toll-like Receptors in the Induction of the Innate Immune Response”. Nature 406.6797 (2000): 782-787.

26. Vix K. "Targeting Macrophage Immunometabolism: Dawn in the Darkness of Sepsis". International Immunopharmacology 58 (2018): 173-185.

27. Kayle D., et al. "Inflammatory Response to Different Toxins in Experimental Sepsis Models". International Journal of Molecular Sciences 20.18 (2019): 1-13.
28. Anthony L., et al. "Biology and Metabolism of Sepsis: Innate Immunity, Bioenergetics, and Autophagy". Surgical Infections 17.3 (2016): 286-293.

29. Jean-Marc C., "Exotoxins and Endotoxins: Inducers of Inflammatory Cytokines". Toxicon 149 (2018): 45-53.

30. Min H., et al. "The Pathogenesis of Sepsis and Potential Therapeutic Targets". International Journal of Molecular Sciences 20.21 (2019): 1-31.

31. Andrew R., et al. "Surviving Sepsis Campaign: International Guidelines for Management of Sepsis and Septic Shock: 2016". Critical Care Medicine 45.3 (2017): 486-552.

32. Jordi R., et al. "Sepsis: A Review of Advances in Management". Advances in Therapy 34.11 (2017): 2393-2411.

33. Danyang L., et al. "Liberal versus Conservative Fluid Therapy in Adults and Children with Sepsis or Septic Shock". Cochrane Database of Systematic Reviews 12 (2018): 1-38.

34. Benjamin C., et al. "Cytokine Storm and Sepsis Disease Pathogenesis”. Seminars in Immunopathology 39.5 (2017): 517-528.

35. Tom VP., et al. "The Immunopathology of Sepsis and Potential Therapeutic Targets". Nature Reviews Immunology 17.7 (2017): 407-420.

36. Yvette L., et al. "Arginine Infusion in Patients with Septic Shock Increases Nitric Oxide Production without Haemodynamic Instability". Clinical Science 128.1 (2015): 57-67.

37. Richard B., et al. "Early Enteral Supplementation with Key Pharmaconutrients Improves Sequential Organ Failure Assessment Score in Critically Ill Patients with Sepsis: Outcome of a Randomized, Controlled, Double-Blind Trial*”. Read Online: Critical Care Medicine | Society of Critical Care Medicine 36.1 (2008): 131-144.

38. Rolando DR., et al. "NutriSim (C) protects against hippocampal neuronal damage induced by ischemia-reperfusion in Mongolian gerbils (Meriones unguiculatus): Morphological and biochemical approach". Journal of Neuroscience and Behavioural Health 2.1(2010): 7-11.

39. Irma VB., et al. "Effect of NutriSim (C) and the Interactive Response of Pro-inflammatory and Anti-inflammatory Cytokines in a Model of Septic Shock Induced by E coli Serotype 0111: B4". American Journal of Biochemistry 4.1 (2014): 6-13. 
40. Erika GR., et al. "Effect of Nutrisim(C) on Endotoxic Shock Induced by Lipopolysaccharide from Escherichia coli: 0111:B4 in Rats: Structural Study of Liver, Kidney and Lung". Journal of Clinical and Experimental Pathology 4.1 (2013): 1-6.

41. Erandis TS., et al. "Beneficial effect of NutriSim(C) against LPS-induced liver injury: Oxidative stress markers and mitochondrial ATP-ase activity". International Journal of Biological Chemistry 7.1 (2013): 38-46.

42. Oscar BQ., et al. "NutriSim (C Diminishes LipopolysaccharideStimulated Anti-inflammatory IL-4 and IL-10 Cytokines and Nitric Oxide". International Journal of Medical Sciences and Health Care 1.8 (2013): 8-12.

43. Erika GR., et al. "NutriSim(C) Diminishes an Endotoxin-stimulated Proinflammatory Cytokine Production". American Journal of Biochemistry 2.6 (2012): 94-97.

44. Peng L., et al. "Amino acids and immune function". British Journal of Nutrition 98.2 (2007): 237-252.

45. Ewa S., et al. "Oxidative changes in the liver, brain and lens of lipopolysaccharide-treated rats". Archives of Medical Research 26 (1995): 109-115.

46. Eugui EM., et al. "Some antioxidants inhibit, in a co-ordinate fashion, the production of tumor necrosis factor-alpha, ILbeta, and IL- 6 by human peripheral blood mononuclear cells". International Immunology 6.3 (1994): 409-22.

47. Barbara L., et al. "L-Arginine reduces lipid peroxidation in patients with diabetes mellitus". Free Radical Biology and Medicine 22.1 (1997): 355-7.

48. Guoyao W., et al. "Arginine nutrition in development, health and disease". Current Opinion in Clinical Nutrition and Metabolic Care 3.1 (2000): 59-66.

49. Thomas R., et al. "Antinociceptive and anti-inflammatory effects of choline in a mouse model of postoperative pain". British Journal of Anaesthesia 105.2 (2010):201-207.

50. Luis FG., et al. "Compuestos nitrogenados de interés en nutrición clínica”. Nutrición Hospitalaria 21.2 (2006):15-29.

51. Hui H., et al. "Effects of dietary lysine restriction on inflammatory responses in piglets". Scientific Reports 8.1 (2018):1-8.

52. Kohsuke H., et al. "Safety assessment of l-lysine oral intake: a systematic review". Amino Acids 51.4 (2019):647-659.
53. Heikki M., et al. "Dietary calcium and magnesium supplements in spontaneously hypertensive rats and isolated arterial reactivity". British Journal of Pharmacology 115.8 (1995): 14551462.

54. Alyssa M., et al. “Biochemistry, Nutrients”. Stat Pearls (2020).

55. Juan 0., et al. "Effects of L-Arginine on the Proliferation of T Lymphocyte Subpopulations". Journal of Parenteral and Enteral Nutrition 25.1 (2001): 23-29.

\section{Assets from publication with us}

- Prompt Acknowledgement after receiving the article

- Thorough Double blinded peer review

- Rapid Publication

- Issue of Publication Certificate

- High visibility of your Published work

Website: https://www.actascientific.com/

Submit Article: https://www.actascientific.com/submission.php Email us: editor@actascientific.com

Contact us: +919182824667 